\title{
Non-Functioning Endocrine Neoplasm
}

National Cancer Institute

\section{Source}

National Cancer Institute. Non-Functioning Endocrine Neoplasm. NCI Thesaurus. Code C94760.

A hormone producing or non-producing endocrine neoplasm, not associated with a hormonal syndrome. 